\title{
Angle Discrimination by Walking in Children
}

\author{
Luigi Cuturi \\ Italian Institute of Technology \\ Genoa, Italy \\ Luigi.Cuturi@iit.it
}

\author{
Giulia Cappagli \\ Italian Institute of Technology \\ Genoa, Italy \\ Giulia.Cappagli@iit.it
}

\author{
Monica Gori \\ Italian Institute of Technology \\ Genova, Italy \\ Monica.Gori@iit.it
}

\begin{abstract}
In primary school, children tend to have difficulties in discriminating angles of different degrees and categorizing them either as acute or obtuse, especially at the first stages of development (6-7 y.o.). In the context of a novel approach that intends to use other sensory modalities than visual to teach geometrical concepts, we ran a psychophysical study investigating angle perception by spatially navigating in space. Our results show that the youngest group of children tend to be more imprecise when asked to discriminate the walking angle of $90 \hat{A}$ r̆, pivotal to learn how to differentiate between acute and obtuse angles. These results are then discussed in terms of the development of novel technological solutions aimed to integrate locomotion in the teaching of geometrical concepts.
\end{abstract}

\section{CCS CONCEPTS}

- Human-centered computing $\rightarrow$ Empirical studies in HCI; Laboratory experiments;

\section{KEYWORDS}

Angle Perception, Locomotion, Self-Motion, Teaching, Geometry

\section{ACM Reference Format:}

Luigi Cuturi, Giulia Cappagli, and Monica Gori. 2017. Angle Discrimination by Walking in Children. In Proceedings of 1st ACM SIGCHI International Workshop on Multimodal Interaction for Education (MIE'17). ACM, New York, NY, USA, 4 pages. https://doi.org/10.1145/3139513.3139516

\section{INTRODUCTION}

The ability to discriminate angles of different degrees is taught during primary school and prepares for learning geometrical concepts of increasing complexity, e.g. trigonometry. For such geometrical concepts, the sensory modality that is mostly used is visual, indeed students are mostly requested to look at and recognize angles of different degree rather than exploring them through the other senses. Generally, children are firstly taught to differentiate between acute and obtuse angles. In this sense, it is fundamental for the teacher to make sure children understood the concept of right angle (i.e. $90^{\circ}$ ) as it represents the boundary between acute and obtuse angles. Most of the research investigating the capability of discriminating angles has focused on visual and proprioceptive [7] or visual and haptic [5] perception of angles but not many studies have employed active locomotion as mean to perceive angles of different degree. In

Permission to make digital or hard copies of part or all of this work for personal or classroom use is granted without fee provided that copies are not made or distributed for profit or commercial advantage and that copies bear this notice and the full citation on the first page. Copyrights for third-party components of this work must be honored.

For all other uses, contact the owner/author(s).

MIE'17, November 13, 2017, Glasgow, UK

(c) 2017 Copyright held by the owner/author(s)

ACM ISBN 978-1-4503-5557-5/17/11.

https://doi.org/10.1145/3139513.3139516

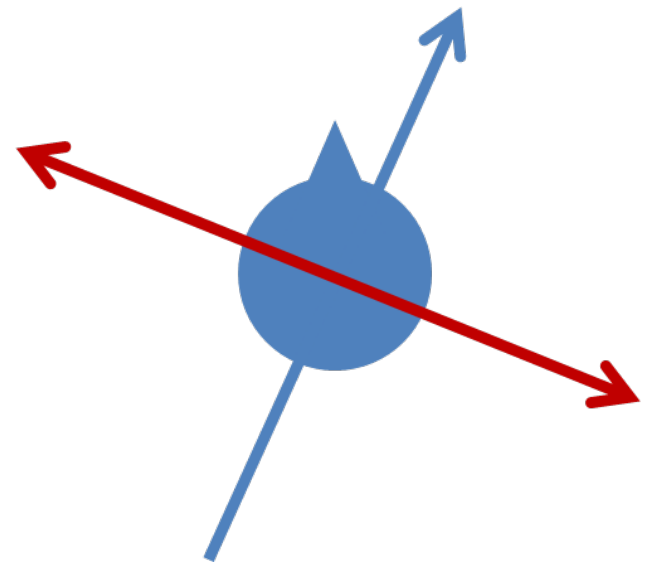

Figure 1: Example of idiosyncratic biases to the right and left (double-headed red arrow). These biases reflect a bias to the right for straight ahead given by combining the overestimation of $90^{\circ}$ to the left and the underestimation of $90^{\circ}$ to the right.

a recent study, Jetzschke and colleagues [4] reported the presence of idiosyncratic biases in adults when requested to indicate whether they perceive to have turned more or less than $90^{\circ}$ in a walking task. Such idiosyncratic biases revealed that biases to the right and to the left are correlated thus indicating the presence of a bias for the straight ahead direction (see red line in Fig. 1).

In this work, our goal is to test whether children of primary scholar show intrinsic biases in perceiving the turning angle of $90^{\circ}$. To this aim, we used a s a similar protocol to the one used by Jetzschke and colleagues [4]. We intend to quantify how accurate and precise children are in discriminating angles of different degree depending on their developmental stage. This information can then be employed to create new methods and technology to teach geometrical concepts as angle discrimination for children of every age.

\section{METHODS}

Forty-two children (age range: 6-11 y.o.) were recruited. In order to make sure they could not see their steps and the floor, participants were blindfolded and additionally provided with a customized silicon bib (see Fig. 2A). The experimenter guided them along two segments (see Fig. 2B), the first one $225 \mathrm{~cm}$ long and the second one $120 \mathrm{~cm}$ long. The angle between the segments was varied on a trial-by-trial basis in the range between $\pm 50^{\circ}$ and $\pm 130^{\circ}$ with a step size of $20^{\circ}$. Each stimulus level was repeated 5 times, leading 


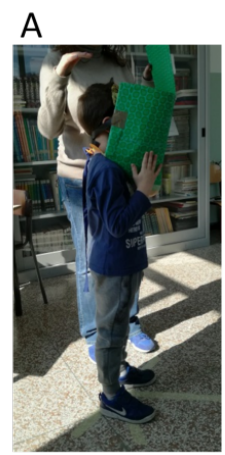

B

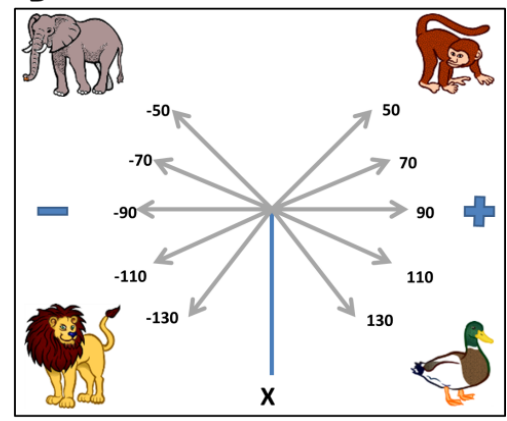

Figure 2: Example subject wearing the customized bib (A). Illustration of the experimental procedure (B): the angle is defined as the aperture of the angle subtended by subject movement. $X$ is the starting position for all movements, the blue segment is the first walked segment and the gray arrows represent the turning angles. The pictured animals represent the landmarks used by subjects to give their response.

to 25 trials per block of trials; each block lasted 30 minutes. Due to the long duration of the experiment and to children difficulty to maintaining a reliable level of awareness, blocks of trials for each side were performed in different days. In order to maintain the same presentation order for each participant, we randomized the order across trials once and used that same order for each participant. Movements to the right (positive sign) and to the left (negative signs) were performed in separate blocks of trials and in separate days to make sure that participants would be able to complete the experiment. Since children might have difficulties in understating the concept of angles and especially the right angle, we employed visual landmarks positioned on the floor to be used as reference for their response (see Fig. 2B). Before each trial, the subject was asked to remove the blindfold and look at the 4 visual landmarks. After being guided along the two segments by the experimenter, subjects were asked to report whether they arrived closer to one landmark or the other. For each block of trials subjects were forced to use the landmarks of the target side, either to the right or to the left. The landmarks were drawings of animals which were part of a story told to the subject in order to make the experiment enjoyable: each participant was told that they were going to explore a forest by being hidden inside a bush (represented by the customized silicon bib); their task was to understand whether they went closer to an animal or the other and report the response to the experimenter. After each trial, the experimenter accompanied the subject back to the start position by making a random path in order to avoid potential feedbacks about performance during the task.

\section{DATA ANALYSIS AND RESULTS}

In order to extrapolate how accurate and precise children performance is, we used an established psychophysical method, that is a psychometric function fitted to the collected data. To this aim, we fit a cumulative Gaussian to the data using the PAL_PFML_Fit routine from the Palamedes toolbox [6] which finds the best fit in a maximum likelihood sense. The mean of the distribution provides a measure of the point of subjective equality (PSE) that is the angle at which the turning angle is equally perceived to be acute or obtuse, thus perceived as a right angle (i.e. $90^{\circ}$ ). Accuracy for each participant is then given by the PSE; any deviation from the right angle represent a bias in the estimate, thus indicating that participants under- or overestimate the right angle. As a measure of precision associated with the estimate we used the SD that is the standard deviation of the fitted psychometric function and indicates variability associated with the estimate (see Fig. 3). Given the high variability in subjects performance and the low number of repetitions per stimulus level, for 53 over 74 measurements, the psychometric fit returned an SD lower than the minimum difference between the stimuli (e.g. see Fig. 3). Therefore, for the abovementioned selected dataset, we replaced the SD to be $20^{\circ}$ and conducted the statistical analysis on these values. In the remaining cases the SD returned a level greater than 20 , therefore we used the original values returned by the fit. We calculated the bias for each participant as the difference between the extrapolated PSE and the right angle (i.e. $90^{\circ}$ ): negative biases indicate underestimation of the right angle whereas positive biases indicate overestimation. In Fig. 4 and Fig. 5, we show averaged biases and SD by grouping children according to three age groups: 6-7, 8-9 and 10-11 y.o. The values of the average bias for each age group are reported in table 1 . Biases are mostly negative for all ages and both for left and right sides. It can be noted that the oldest group of participants (10-11 y.o.) shows biases that tend to be on the opposite direction with a reduced bias for the angles to the left compared to the angles to the right side. However, a mixed ANOVA analysis did not reveal any significant effect given by the age group $(\mathrm{F}=0.3, \mathrm{p}=0.7)$, the side of movement $(\mathrm{F}=0.2, \mathrm{p}=$ $0.6)$ and the interaction of these factors $(F=0.8, p=0.4)$. Regarding precision, it can be seen that the younger the children group, the higher is the SD, thus indicating lower precision at this developmental stage as previously observed in other studies investigating multisensory perception across development [3]. This result was confirmed by a mixed ANOVA that shows a significant effect (alpha $=0.05)$ given by the age group $(\mathrm{F}(2,39)=3.8, \mathrm{p}=0.03)$ whilst there was no significant effect given by the side of movement $(\mathrm{F}(1,39)=$ $2.3, \mathrm{p}=0.1)$ and the interaction between the two factors $(\mathrm{F}(2,39)=$ $0.3, p=0.7)$. Post-hoc analysis (t-tests) on the variability data for both sides of movement reveal a tendency towards a significant difference between the age groups of 6-7 and 8-9 y.o. $(\mathrm{t}=2.0, \mathrm{p}=$ $0.05)$ and the age groups of 6-7 and 10-11 y.o. $(t=1.8, p=0.07)$. We did not observe any trends for a difference between the age groups of 8-9 and 10-11 y.o. However, these tests are not corrected for multiple comparison, therefore further analysis and data collection might be needed. As in the work of Jetzschke and colleagues [4], we ran a correlation analysis to test whether the perceived angle of 90 Âr to the right and to the left are combined in a bias for straight ahead. We did not observe any significant correlation for all age groups (Pearson correlation: 6-7 y.o., $\mathrm{R}=0.42$, $\mathrm{p}=0.19$; 8-9 y.o., $\mathrm{R}$ $=-0.29, \mathrm{p}=0.2 ; 10-11$ y.o., $\mathrm{R}=-0.21, \mathrm{p}=0.52$.), thus indicating that children do not integrate biases for the left and right side in a bias for straight ahead. 

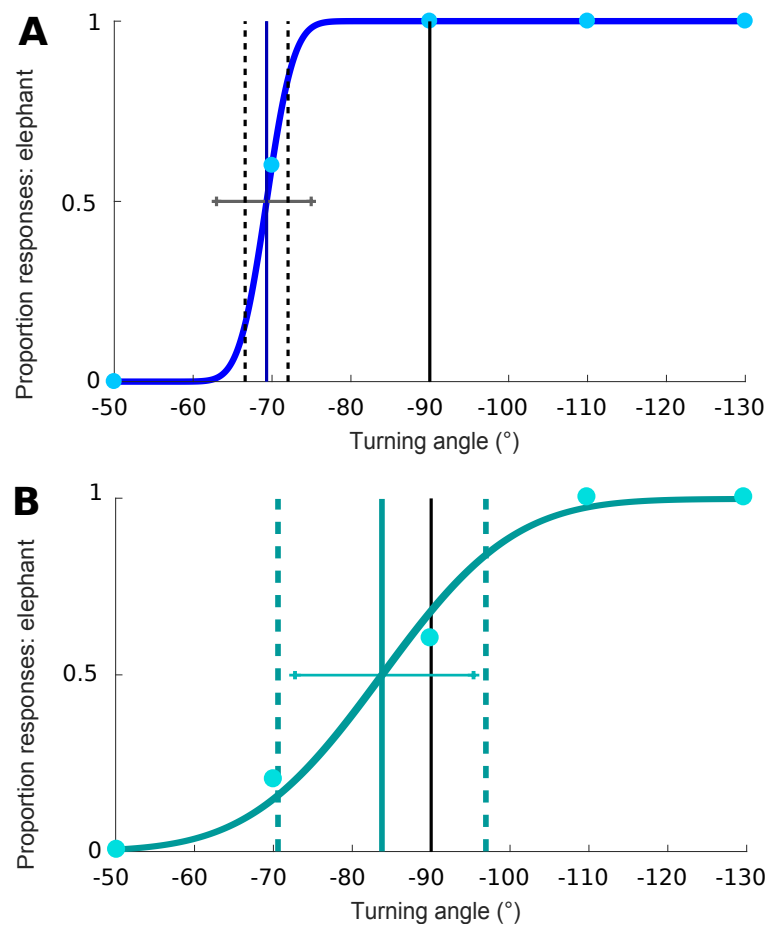

Figure 3: Psychometric function for two individual subjects aged 8 y.o. (A) and 9 y.o. (B) for movements to the left. The light colored dots represent the proportion of responses "elephant" for each stimulus level. The unbiased estimate is represented by the black vertical line centered at $-90^{\circ}$. The PSE is indicated by the solid colored vertical line. The vertical dotted lines represent the PSE +/- SD that is the variability associated with the estimate. In A, the PSE shift shows an underestimation of the $90^{\circ}$ turning angle corresponding to a negative bias of $-20.7^{\circ}$ with a SD of $2.7^{\circ}$. In B, the PSE shift shows an underestimation of the $90^{\circ}$ turning angle corresponding to a negative bias of $6.2^{\circ}$ with a SD of $13^{\circ}$. The horizontal line represents $95 \%$ confidence interval.

Table 1: Table showing the bias for each age group.

\begin{tabular}{|c|c|c|c|c|}
\cline { 3 - 5 } \multicolumn{2}{c|}{} & \multicolumn{3}{c|}{ Age (y.o.) } \\
\cline { 3 - 5 } \multicolumn{2}{c|}{} & $6-7$ & $8-9$ & $10-11$ \\
\hline \multirow{2}{*}{ Side } & Right & $-4,2$ & -8 & $-7,4$ \\
\cline { 2 - 5 } & Left & $-8,4$ & -6 & $-1,2$ \\
\hline
\end{tabular}

\section{CONCLUSIONS}

This work aimed to disclose the perceptual basis behind angle perception by walking in children. Our results show that perception of the right angle is biased across all ages. These biases do not correlate between sides in spite of previous observations in adults. This finding indicates that children need more time to integrate their perceived turning angles to the two sides into one more complex spatial representation that takes both sides in account.

Interestingly, the youngest group of children show lower precision in perceiving angles to the right and left compared to their older

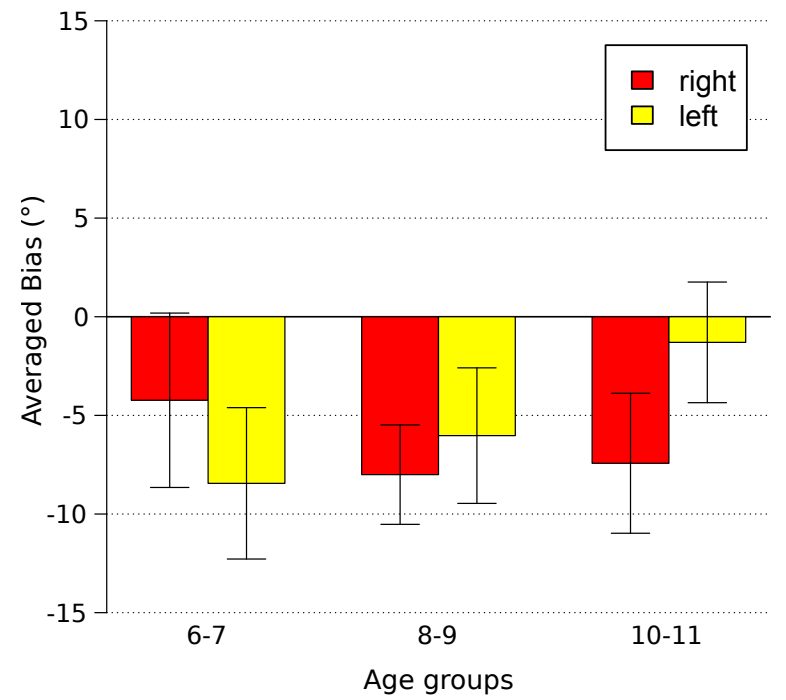

Figure 4: Biases of the perceived $90^{\circ}$ turning angle to the right and to the left. Negative biases indicate underestimation and positive biases represent overestimation of the $90^{\circ}$ turning angle. Error bars represent standard error.

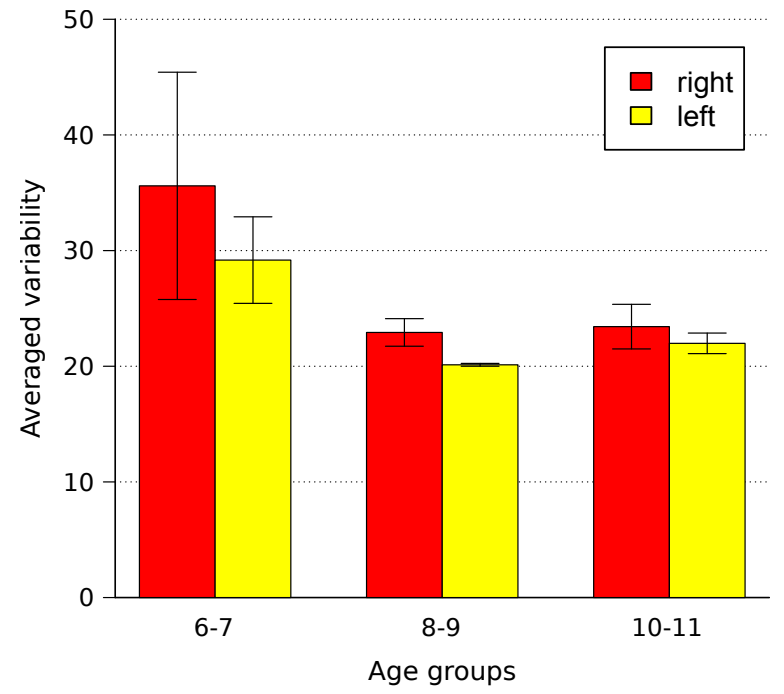

Figure 5: Precision associated with the perceived turning angle of $90^{\circ}$ to the right and to the left. Precision is represented as the variability (SD of the psychometric function) in degrees $\left(^{\circ}\right)$ averaged across subjects. Error bars represent standard error.

peers. Children of 6-7 years old seem to need a big turn (between $20^{\circ}$ and $30^{\circ}$ ) in order to being able to discriminate between acute and obtuse angles. Although these values are based on systematic psychophysical measurements additional analysis are needed in order to be definitive and provide the proper quantification of precision depending on children age. An issue that is often encountered in research in developmental science is related to the high variability 
in children performance. Provided the right analysis, this result would be of great importance as it could be used in relationship to the potential employment of locomotion in teaching geometry and in particular discrimination of angle apertures with respect to the right angle.

The results presented here are valuable inputs for the WeDraw project (www.wedraw.eu), a two-year project with the scope of integrating multisensory teaching tools to the current teaching approach in primary school. The task presented here provides information that may be valuable for teachers. Knowing that tracing angles by walking is biased in most of children of primary school provides fundamental insights in the use of motor exercises to teach angles of different aperture. Teachers may adapt the task used in this experiment by simplifying it (e.g. by using the greater apertures only) to test whether specific children have biases in understanding the $90^{\circ}$ turn. Overall, the main aim of this work is to provide the scientific basis for the development of new technological solutions to be used to teach concepts as angle aperture discrimination and comprehension of the right angle. The integration of our results with the technology developed by the project partners (e.g. EyesWeb, [2], [1], [8]) and their application to use commercial devices as motion sensors (e.g. Kinect, a motion sensing input device by Microsoft) can be applied to introduce new sets of exercises aimed to teach geometrical concepts related to angles. In this sense, our results provide the psychophysical basis to develop serious games and exercises that use locomotion in space to track angle aperture and provide primary school teachers with science-based tools for teaching geometrical concepts.

\section{ACKNOWLEDGMENTS}

We thank Elisa Freddi for assistance with data collection. This work was supported by the European WeDraw project (ICT-22-2016 Technologies for Learning and Skills, under Grant Agreement No. 732391).

\section{REFERENCES}

[1] Antonio Camurri, Paolo Coletta, Giovanna Varni, and Simone Ghisio. 2007. Developing multimodal interactive systems with EyesWeb XMI. In Proceedings of the 7 th international conference on New interfaces for musical expression - NIME '07. 305. https://doi.org/10.1145/1279740.1279806

[2] Antonio Camurri, Barbara Mazzarino, and Gualtiero Volpe. 2004. Analysis of expressive gesture: The eyesweb expressive gesture processing library. In Gesturebased Communication in Human-Computer Interaction, LNAI 2915 (2004), 460-467. https://doi.org/10.1007/978-3-540-24598-8_42

[3] Monica Gori, Michela Del Viva, Giulio Sandini, and David C. Burr. 2008. Young Children Do Not Integrate Visual and Haptic Form Information. Current Biology 18, 9 (2008), 694-698. https://doi.org/10.1016/j.cub.2008.04.036 arXiv:http://dx.doi.org/10.1121/1.4906832

[4] Simon Jetzschke, Marc O. Ernst, Alessandro Moscatelli, and Norbert Boeddeker. 2016. Going round the bend: Persistent personal biases in walked angles. Neuroscience Letters jo (2016). https://doi.org/dx.doi.org/10.1016/j.neulet.2016.01.026 arXiv:arXiv: $1011.1669 \mathrm{v} 3$

[5] Stephen Lakatos and Lawrence E. Marks. 1998. Haptic underestimation of angular extent. Perception 27, 6 (1998), 737-754.

[6] N Prins and F A A Kingdon. 2009. Palamedes: Matlab routines for analyzing psychophysical data. Palamedes: matlab routines for analyzing psychophysical data (2009).

[7] Johanna Reuschel, Knut Drewing, D. Y P Henriques, Frank Rösler, and Katja Fiehler. 2010. Optimal integration of visual and proprioceptive movement information for the perception of trajectory geometry. Experimental Brain Research 201, 4 (2010), 853-862. https://doi.org/10.1007/s00221-009-2099-4 arXiv:NIHMS150003

[8] Gualtiero Volpe, Paolo Alborno, Antonio Camurri, Paolo Coletta, Simone Ghisio, Maurizio Mancini, Radoslaw Niewiadomski, and Stefano Piana. 2016. Designing Multimodal Interactive Systems using EyesWeb XMI.. In SERVE@ AVI. 49-56. 